\title{
Attenuation of Experimental Autoimmune Uveitis in Lewis Rats by Betaine
}

\author{
Yuna Choi ${ }^{1}$, Kyungsook Jung ${ }^{2}$, Hyo Jin $\mathrm{Kim}^{3}$, Jiyoon Chun ${ }^{1}$, Meejung Ahn ${ }^{4}$, Youngheun Jee ${ }^{1}$, \\ Hyun Ju Ko ${ }^{1}$, Changjong Moon ${ }^{5}$, Hiroshi Matsuda ${ }^{6}$, Akane Tanaka ${ }^{6}$, \\ Jeongtae $\mathrm{Kim}^{7 *}$ and Taekyun Shin ${ }^{1 *}$ \\ ${ }^{1}$ College of Veterinary Medicine and Veterinary Medical Research Institute, Jeju National University, Jeju 63243, \\ ${ }^{2}$ Functional Biomaterials Research Center, Korea Research Institute of Bioscience and Biotechnology, Jeongeup 56212, \\ ${ }^{3}$ Department of Food Bioengineering, Jeju National University, Jeju 63243, ${ }^{4}$ Department of Animal Science, \\ College of Life Science, Sangji University, Wonju 26339, ${ }^{5}$ Department of Veterinary Anatomy and Animal Behavior, \\ College of Veterinary Medicine and BK21 Plus Project Team, Chonnam National University, Gwangju 61186, Korea, \\ ${ }^{6}$ Laboratory of Veterinary Molecular Pathology and Therapeutics, Division of Animal Life Science, Graduate School, \\ Institute of Agriculture, Tokyo University of Agriculture and Technology, Tokyo 183-8509, Japan, \\ ${ }^{7}$ Department of Anatomy, Kosin University College of Medicine, Busan 49267, Korea
}

\begin{abstract}
Experimental autoimmune uveitis (EAU) is an animal model of human autoimmune uveitis that is characterized by the infiltration of autoimmune T cells with concurrent increases in pro-inflammatory cytokines and reactive oxygen species. This study aimed to assess whether betaine regulates the progression of EAU in Lewis rats. EAU was induced via immunization with the interphotoreceptor retinoid-binding protein (IRBP) and oral administration of either a vehicle or betaine $(100 \mathrm{mg} / \mathrm{kg})$ for 9 consecutive days. Spleens, blood, and retinas were sampled from the experimental rats at the time of sacrifice and used for the T cell proliferation assay, serological analysis, real-time polymerase chain reaction, and immunohistochemistry. The T cell proliferation assay revealed that betaine had little effect on the proliferation of splenic T cells against the IRBP antigen in an in vitro assay on day 9 post-immunization. The serological analysis showed that the level of serum superoxide dismutase increased in the betainetreated group compared with that in the vehicle-treated group. The anti-inflammatory effect of betaine was confirmed by the downregulation of pro-inflammation-related molecules, including vascular cell adhesion molecule 1 and interleukin- $1 \beta$ in the retinas of rats with EAU. The histopathological findings agreed with those of ionized calcium-binding adaptor molecule 1 immunohistochemistry, further verifying that inflammation in the retina and ciliary bodies was significantly suppressed in the betaine-treated group compared with the vehicle-treated group. Results of the present study suggest that betaine is involved in mitigating EAU through anti-oxidation and anti-inflammatory activities.
\end{abstract}

Key words: Anti-inflammation, Anti-oxidation, Betaine, Experimental autoimmune uveitis, Retina

Submitted May 1,2021, Revised August 11,2021,

Accepted August 12,2021

* To whom correspondence should be addressed. Taekyun Shin, TEL: 82-64-754-3363, FAX: 82-64-756-3354 e-mail: shint@jejunu.ac.kr

Jeongtae Kim, TEL: 82-51-990-6412, FAX: 82-51-241-5458

e-mail:kimjt78@kosin.ac.kr

\section{INTRODUCTION}

Autoimmune uveitis in humans is the leading cause of visual disorders encompassing chronic inflammatory conditions and is considered a vision-threatening disease [1]. Similar to other autoimmune diseases, such as autoimmune encephalomyelitis [2], autoimmune myocarditis [3], and autoimmune neuritis [4], experimental autoimmune uveitis (EAU), which is an animal model of human autoimmune uveitis, is a T cell-mediated disease [5].
Copyright (c) Experimental Neurobiology 2021. www.enjournal.org
This is an Open Access article distributed under the terms of the Creative Commons Attribution Non-Commercial License (http://creativecommons.org/licenses/by-nc/4.0) which permits unrestricted non-commercial use, distribution, and reproduction in any medium, provided the original work is properly cited. 
The homing of autoreactive T cells and infiltration of inflammatory cells, such as monocytes, trigger uveitis and retinitis in EAU [6]. The retina is damaged from inflammation with the activation of glial cells that undergoes oxidative stress [7].

Betaine, also called trimethylglycine $\left(\mathrm{C}_{5} \mathrm{H}_{11} \mathrm{NO}_{2}\right)$, is an alkaloid and nontoxic natural substance from Fructus lycii, and a representative antioxidant substance [8]. Betaine improves age-related inflammation in rats through nuclear factor- $\kappa \mathrm{B}$ involvement via nuclear factor-inducing kinase/I kappa B kinase and mitogen-activated protein kinases [9], human cardiovascular disease by suppressing inflammatory cytokines, including interleukin (IL)-6 and tumor necrosis factor- $\alpha$ (TNF- $\alpha$ ) [10], and dextran sulfate sodiuminduced colon tumorigenesis [11]. In addition, betaine prevented pathological angiogenesis/neovascularization in rats with diabetic retinitis [12] and protected retinal ganglion cells to increase visual acuity in an animal model of glaucoma [13]. However, there is little known about the precise mechanisms underlying the effects of betaine in uveitis.

In this study, the efficacy of betaine in relieving EAU was evaluated. We investigated the anti-inflammatory effect of betaine in EAU based on histopathological examination and cytokine measurements. Furthermore, the specific mechanism of betaine as an antioxidant was assessed in rats with EAU.

\section{MATERIALS AND METHODS}

\section{Animals}

Both sexes of Lewis rats (7 9 weeks old; Orient Bio Inc., Gyeonggi-do, Korea) were housed in our facility under laboratory conditions (12-h light/dark cycle, temperature $23 \pm 2^{\circ} \mathrm{C}$ ). All experimen- tal procedures were performed following the Guidelines for the Care and Use of Laboratory Animals of Jeju National University (permission number: 2020-0012). All animal protocols conformed to international laws and NIH policies, including the Care and Use of Laboratory Animals (NIH publication no. 85-23, 1985, revised 1996).

\section{Induction of EAU}

The rats were immunized with $200 \mu \mathrm{l}$ of a mixed emulsion composed of an equal volume of bovine interphotoreceptor retinoid-binding protein (IRBP) (1 mg/ml; PTARSVGAADGSSWEGVGVVPDV, Komabiotech, Seoul, Republic of Korea) and Freund's complete adjuvant (CFA) supplemented with $1 \mathrm{mg} / \mathrm{mL}$ Mycobacterium tuberculosis H37Ra (Difco Laboratories Inc., Detroit, MI, USA) on the footpads of their hind limbs.

\section{Experimental groups}

To assess the effects of betaine (Fig. 1A) on EAU, four experimental groups were designated as follows: normal control $(n=8)$; CFA control $(n=8)$; EAU+Vehicle $(n=8)$; and EAU+Betaine $(n=8)$. The dose in the treatment to test the therapeutic effect of betaine (100 $\mathrm{mg} / \mathrm{kg}$ body weight/day, B2629, Sigma-Aldrich, St. Louis, MO, USA) was selected based on a previous study [14]. The rats were orally treated 9 with betaine from day 0 post-immunization until day 9 post-immunization.

\section{Tissue preparation}

The rats were sacrificed under deep anesthesia via $\mathrm{CO}_{2}$ gas inhalation on day 9 post-immunization. The tissues for the histopathological examination were embedded in paraffin wax and sectioned
A

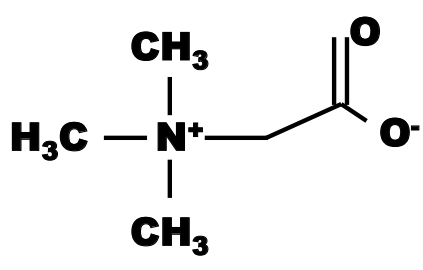

Betaine
B

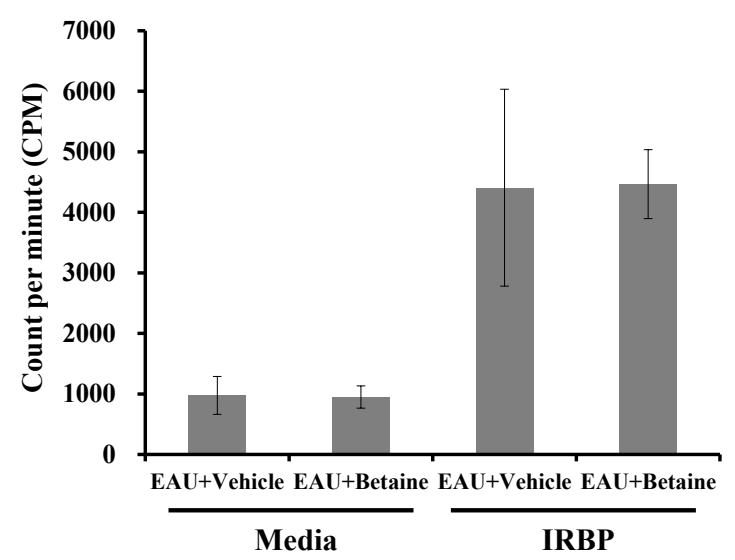

Fig. 1. Chemical structure and effects of betaine on the interphotoreceptor retinoid-binding protein (IRBP)-reactive T cell response. (A) The chemical structure of betaine (Formula: $\left.\left(\mathrm{CH}_{3}\right)_{3} \mathrm{~N}^{+} \mathrm{CH}_{2} \mathrm{COO}\right)^{-}$. (B) Potential inhibitory effects on the T cell proliferative response to the antigen (IRBP) were evaluated in triplicate wells for each experiment $(\mathrm{n}=5)$. Betaine did not affect immunomodulation. 
Table 1. Primer characteristics for real-time polymerase chain reaction

\begin{tabular}{lll}
\hline Primer & \multicolumn{1}{c}{ Forward sequence } & \multicolumn{1}{c}{ Reverse sequence } \\
\hline IL- $1 \beta$ & CCC TGC AGC TGG AGA GTG TGG & TGT GCT CTG CTT GAG AGG TGC \\
TNF- $\alpha$ & CGT CGT AGC AAA CCA CCA AG & CAC AGA GCA ATG ACT CCA AA \\
iNOS & CAG CGC ATA CCA CTT CAG C & ACC ATC GAG CAT CCC AAG \\
COX-2 & CGG AGG AGA AGT GGG GTT TA & TGG GAG GCA CTT GCG TTG AT \\
CAT & CCA CGA GGG TCA CGA ACT GT & CTC CTA TTG CCG TCC GAT TC \\
SOD1 & GGC CAC ACC GTC CTT TCG & CGG TCC AGC GGA TGA AGA \\
SOD2 & TAA GCG TGC TCC CAC ACA TC & ATC AGG ACC CAC TGC AAG GA \\
SOD3 & TGC AGA CTG CGT GCA TCT C & GCG ACA CGC ACT CCA AAG A \\
GAPDH & GGG GGC TCT CTG CTC CTC CC & CGG CCA AAT CCG TTC ACA CCG \\
\hline Primer & Catalog No. & Manufacture \\
\hline Serpina3n & qRnoCID0005765 & Bio-rad, CA, USA \\
VCAM1 & qRnoCID0005077 & Bio-rad, CA, USA \\
\hline
\end{tabular}

IL-1 $\beta$, interleukin- $1 \beta$; TNF- $\alpha$, tumor necrosis factor-alpha; iNOS, inducible nitric oxide synthase; COX-2, cyclooxygenase-2; CAT, catalase; SOD, superoxide dismutase; GAPDH, glyceraldehyde 3-phosphate dehydrogenase; VCAM1, vascular cell adhesion molecule 1.

with a microtome (RM 2135; Leica, Nussloch, Germany) to a thickness of $5 \mu \mathrm{m}$ and stained with hematoxylin and eosin. Blood and retinas were stored at $-80^{\circ} \mathrm{C}$ for the serum analysis and realtime polymerase chain reaction (PCR) analysis.

\section{T cell proliferation assay}

Spleen mononuclear cells from the animals in each group were dissociated and suspended as described in our previous study [15]. Then, $10 \mu \mathrm{g} / \mathrm{ml}$ IRBP (final concentration) was added to the wells. After $48 \mathrm{~h}$ of stimulation with IRBP, the cells were incubated in 1 $\mu \mathrm{Ci}$ of ${ }^{3} \mathrm{H}$-methylthymidine (specific activity $42 \mathrm{Ci} / \mathrm{mmol}$; Amersham, Arlington Heights, IL, USA) for $18 \mathrm{~h}$. Then, the cells were harvested to measure thymidine incorporation.

\section{Serological analysis}

The rats were sacrificed on the sampling date, and blood was collected through the heart. Whole-blood samples were separated into serum and blood cells using a centrifuge (VS-5500CFN; Vision Scientific, Daejeon, Republic of Korea). Superoxide dismutase (SOD) activity in the serum was evaluated using a SOD kit (ab65354; Abcam, Cambridge, UK).

\section{Immunohistochemistry}

Immunohistochemistry was performed using the same protocol as that described in our previous study [16]. The primary antibodies including ionized calcium-binding adapter moleculel (Iba1) (1:1,000; 019-19741, Wako Pure Chemical Industries, Ltd., Osaka, Japan), CD68 (ED1; 1:800; MCA341, Serotec, Kidlington, UK), and glutamine synthetase (GS) (1:5,000; MAB302, Chemicon International, Temecula, CA, USA) were used as marker for microglia, macrophage and Müller cell, respectively.

\section{Real-time PCR}

Total RNA in the eyeballs in all groups ( $\mathrm{n}=5$ per group) was isolated with TRIzol RNA Isolation Reagent (Life Technologies, Thermo Fisher Scientific, Carlsbad, CA, USA), and cDNA was prepared using CellScript ${ }^{\mathrm{TM}}$ All-in-One 5X First Standard cDNA Synthesis Master Mix (CellSafe, Gyeonggi-do, Republic of Korea). The primer information is listed in Table 1. PCR was performed with a MIC cycler (BMS, Queensland, Australia) using $2 \times$ SYBR Green (PhileKorea, Seoul, Republic of Korea) and the following program: 55 cycles of denaturation $\left(5 \mathrm{~s}, 95^{\circ} \mathrm{C}\right)$, annealing $(20 \mathrm{~s}$, $\left.60^{\circ} \mathrm{C}\right)$, and extension $\left(10 \mathrm{~s}, 72^{\circ} \mathrm{C}\right)$.

\section{Western blot analysis}

Western blot analysis was performed by the same protocol as that described in our previous study [16]. The primary antibodies including Kelch-like ECH-associated protein 1 (Keap1) (1:1,000; ab119403, abcam, MA, USA), and Nuclear factor erythroid-2-related factor 2 (Nrf2) (1:1,000; sc-722, Santa cruz, CA, USA).

\section{Statistical analysis}

All measurements are reported as the average of three independent experiments. All values are presented as the mean \pm standard error of the mean (SEM). The results were analyzed using oneway analysis of variance followed by the Student-NewmanKeuls post-hoc test for multiple comparisons. A p-value $<0.05$ was considered to indicate significance. Immunostaining was analyzed semi-quantitatively based on the positive areas in the photographs using Image software (National Institutes of Health, Bethesda, MD, USA). EAU was histopathologically evaluated using a method modified from a previous study [17]. Antibody-positive areas were measured as follows: (1) three different sections from each 
rat ( $\mathrm{n}=3$ animals per group) were used; then, (2) the percentage of the stained area $[($ positive area/total area $) \times 100(\%)]$ was calculated. The total area included all layers of the retina. These results are presented as the mean \pm SEM.

\section{RESULTS}

\section{Betaine had no immunomodulatory function in EAU}

The $\mathrm{T}$ cell proliferation assay was performed to determine whether betaine affected the proliferation of IRBP-specific T cells (Fig. 1B). No significant changes were observed between the EAUinduced groups in medium only and those that were IRBP-stimulated (medium only, $\mathrm{p}>0.05$ vs. EAU+Vehicle; IRBP stimulation, $\mathrm{p}>0.05 \mathrm{vs}$. EAU+Vehicle). These data indicate that betaine was not involved with IRBP-specific T cells or their auto-reactivity.

\section{Betaine upregulated serum SOD level in EAU}

We evaluated oxidative damage in the serum, using SOD as a marker of oxidative modification. No significant difference was observed between the normal and CFA groups. SOD activity decreased significantly in the EAU+Vehicle group, compared to levels in the normal control and CFA groups. Betaine treatment significantly restored the level of SOD activity to that of the normal control and CFA groups (Fig. 2). This result indicates that the betaine treatment suppressed oxidative stress in rats with EAU.

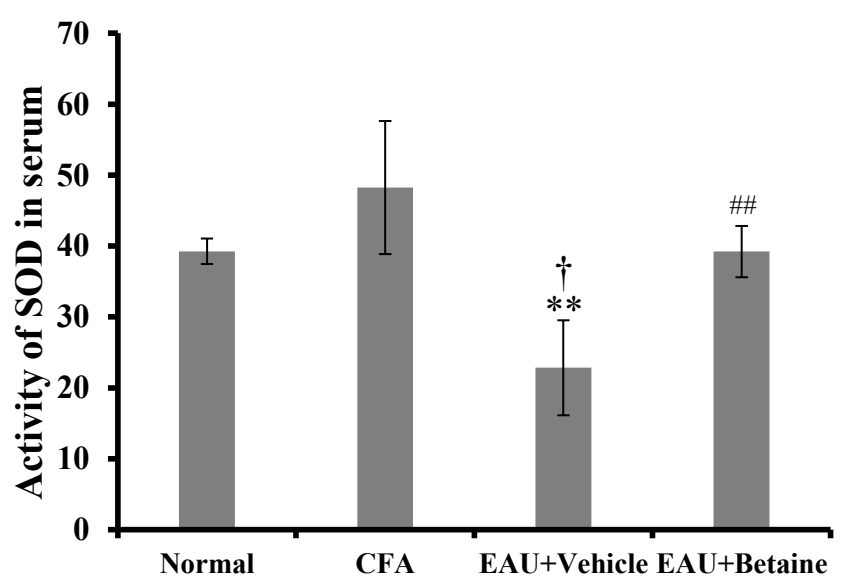

Fig. 2. Superoxide dismutase (SOD) activity in the serum of experimental autoimmune uveitis (EAU)-induced rats treated with or without betaine. SOD activity in the EAU+Vehicle group decreased significantly compared to that in the normal and Freund's complete adjuvant (CFA) groups. However, SOD activity was maintained at the normal level in the betainetreated group (EAU+Betaine). ${ }^{* *} \mathrm{p}<0.01 \mathrm{vs}$. normal control; ${ }^{\dagger} \mathrm{p}<0.05 \mathrm{vs}$. CFA; ${ }^{* \#} \mathrm{p}<0.01$ vs. EAU+Vehicle.
Betaine reduced the infiltration of Iba1-positive cells in the ciliary bodies and retinas of EAU-induced rats

The ciliary body is the main inflammatory cell infiltration site because of the abundance of blood vessels [18]. Only a few roundtype cells were detected in the ciliary bodies in the normal and CFA groups (Fig. 3A, 3B), whereas the infiltration of some roundtype cells was confirmed in the EAU-induced groups (arrows in Fig. 3C, 3D). The normal (Fig. 3E) and CFA (Fig. 3F) groups consistently displayed similar results to those observed for Ibal immunoreactivity. Ibal-positive immunoreactivity increased in the EAU+Vehicle and EAU+Betaine groups (arrowheads in Fig. 3G, $3 \mathrm{H})$. However, the number of Ibal-positive cells decreased significantly in the EAU+Betaine group compared to the EAU+Vehicle group (Fig. 3I). We also analyzed the localization of ED1 as a further approach to evaluate the precise location of inflammatory cell infiltration in the ciliary body. ED1-positive cells were rarely detected in the normal (Fig. 3G) and CFA (Fig. 3K) groups. By contrast, numerous ED1-positive cells were detected in the $\mathrm{EAU}+$ Vehicle and EAU+Betaine groups (double arrowheads in Fig. 3L, 3M). A semi-quantitative analysis of the number of ED1positive cells confirmed that the betaine treatment suppressed the infiltration of inflammatory cells in the ciliary bodies of EAUinduced rats.

Next, we investigated histopathological changes in the retina (Fig. 4). A few inflammatory cells were detected in retinas with EAU, but not in normal and CFA rat retinas (Fig. 4A 4D). The lesions were scored histopathologically according to the severity of EAU [17], revealing relief of retinal inflammation (Fig. 4E). Microglial and Müller cell activation indicating retinal inflammation was confirmed based on Ibal (Fig. 4F 4I) and GS immunoreactivity (Fig. $4 \mathrm{~K} \sim 4 \mathrm{~N}$ ), respectively. The localization of Ibal in microglia was very rare in the normal and CFA groups (arrowheads in Fig. $4 \mathrm{~F}, 4 \mathrm{G}$, respectively). The activation of microglia was inhibited in EAU rats (arrowheads in Fig. $4 \mathrm{H}$ ) by the betaine treatment (Fig. 4I, 4J). The GS-positive immunoreactivity result was similar to that of Ibal in the retina (Fig. $4 \mathrm{~K} \sim 4 \mathrm{~N}$ ). Activated Müller cells in the EAU+Vehicle group had lower GS-immunoreactivity levels (Fig. 4O).

\section{Betaine suppressed adhesion molecule and pro-inflammatory mediators in EAU}

Next, we examined adhesion molecule expression using realtime PCR (Fig. 5A). A sharp decrease in the vascular cell adhesion molecule 1 (VCAM1) mRNA level in the EAU+Betaine group was observed ( $p<0.05$ vs. EAU+Vehicle). The mRNA levels of Serpina3n, interleukin-1 $\beta$ (IL-1 $\beta$ ), tumor necrosis factor-alpha (TNF- $\alpha$ ), inducible nitric oxide synthase (iNOS) and cyclooxygen- 


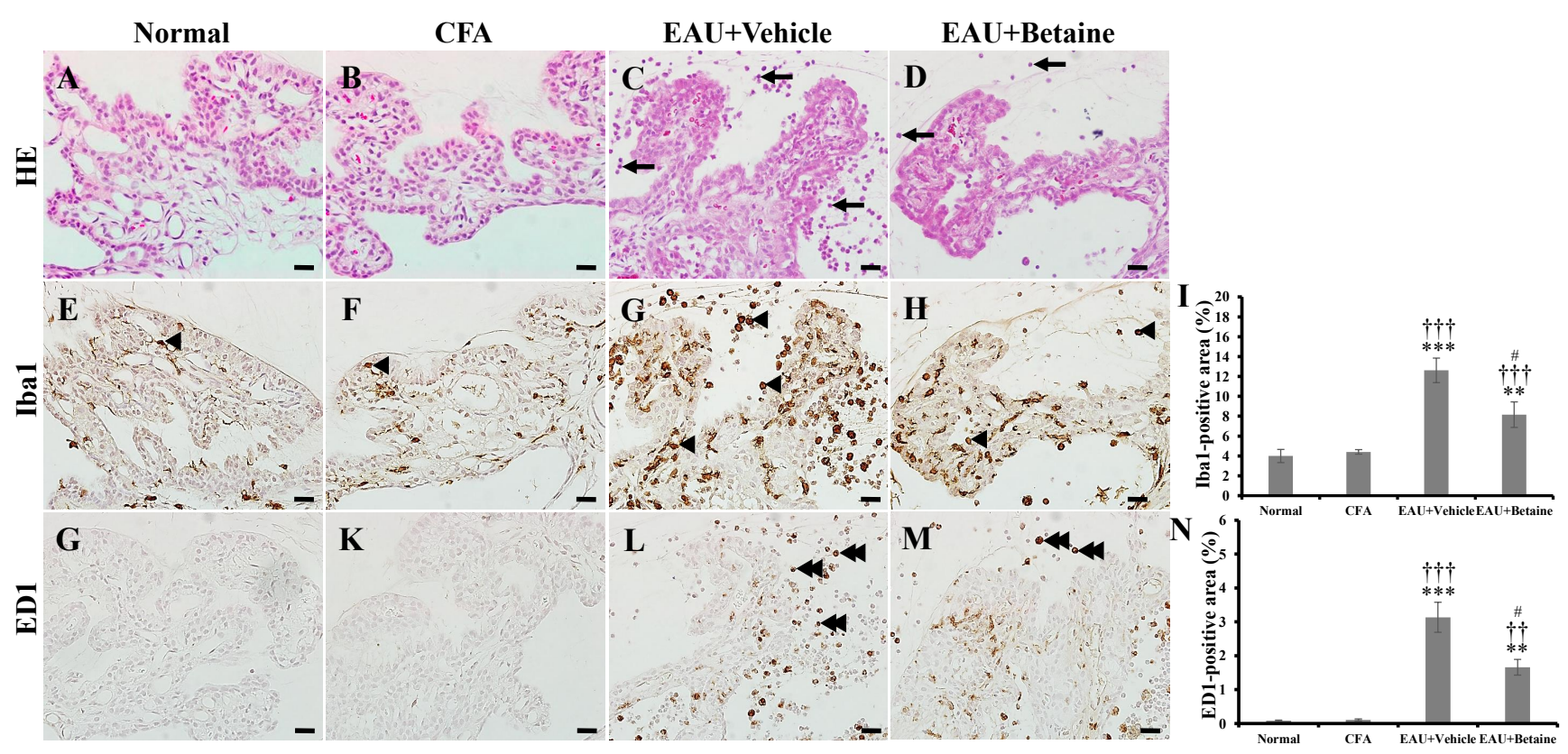

Fig. 3. Histopathological examination of ciliary bodies in the normal (A, E, G), CFA (B, F, K), EAU+Vehicle (C, G, L), and EAU+Betaine (D, H, M) groups. Some rounded cells (arrows in C, D) had infiltrated around the ciliary body in an EAU-induced rat (C, D) but not in normal (A) or CFA (B) rats. An ionized calcium-binding adapter molecule 1 (Ibal)-positive immunoreaction was observed in the ciliary body (arrowheads in E, F, G, H). (I) A significant increase in the Iba1-positive area was confirmed using semi-quantitative analysis. Although the ED1-positive immunoreaction was localized at the infiltrated round cells (double arrowheads in L, M) in the EAU-induced rats, the ciliary bodies of rats in the normal and CFA groups had no ED1positive cells. (N) The ED1-positive area decreased in response to betaine treatment. Scale bars, $50 \mu \mathrm{m} .{ }^{* *} \mathrm{p}<0.01 ;{ }^{* * *} \mathrm{p}<0.001 \mathrm{vs}$. normal control; ${ }^{\dagger \dagger} \mathrm{p}<0.01$; p $<0.001$ vs. CFA; ${ }^{*} \mathrm{p}<0.05$ vs. EAU+Vehicle.

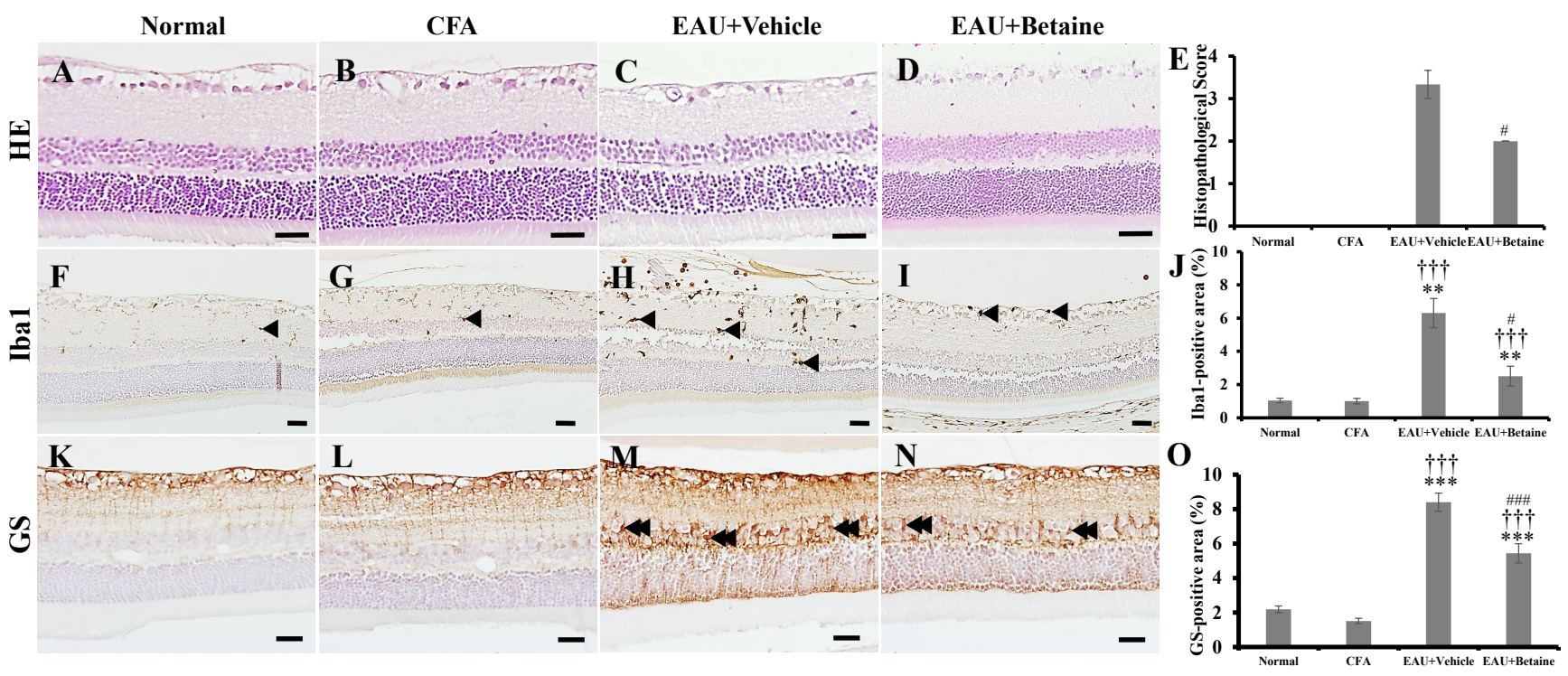

Fig. 4. Histopathological examination of retinas in the normal (A, F, K), CFA (B, G, L), EAU+Vehicle (C, H, M), and EAU+Betaine (D, I, N) groups. The retinal inflammation was difficult to be clearly distinguished in the HE staining (A D). Iba1 immunohistochemistry was performed to reveal clear retinal inflammation. (E) The bar graph displays the comprehensive results of hematoxylin and eosin and Ibal staining. Some Ibal-positive microglia (arrowheads in F, G) were confirmed in the normal (F) and CFA (G) groups. An increased number of Ibal-positive ramified microglia (arrowheads in $\mathrm{H}, \mathrm{I})$ were detected in the EAU-induced retina (H, I). (J) The bar graph displays the results of semi-quantitative analysis. The activation of Müller cells was confirmed via immunohistochemical staining using glutamine synthetase (GS), a Müller cell marker (double arrowhead in M, N), compared to the normal (K) and CFA (L) groups. (O) A significant increase in the GS-positive area was observed in the EAU+Betaine group compared with the EAU+Vehicle group. Scale bars in (A, B, C, D, F, G, H, I), $50 \mu \mathrm{m}$. Scale bars in (K, L, M, N), $20 \mu \mathrm{m} .{ }^{* *} \mathrm{p}<0.01 ;{ }^{* * *} \mathrm{p}<0.001$ vs. normal control; ${ }^{\dagger \dagger t} \mathrm{p}<0.001 \mathrm{vs}$. CFA; ${ }^{*} \mathrm{p}<0.05 ;{ }^{* \# *} \mathrm{p}<0.001$ vs. EAU + Vehicle. 
A

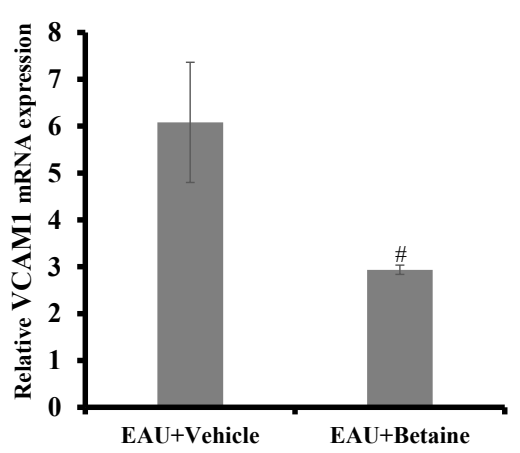

B

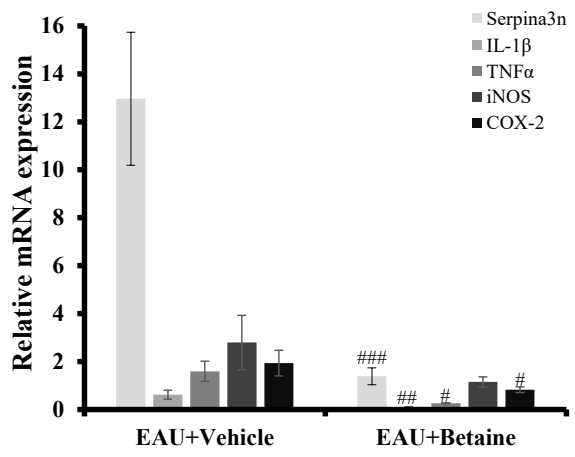

C

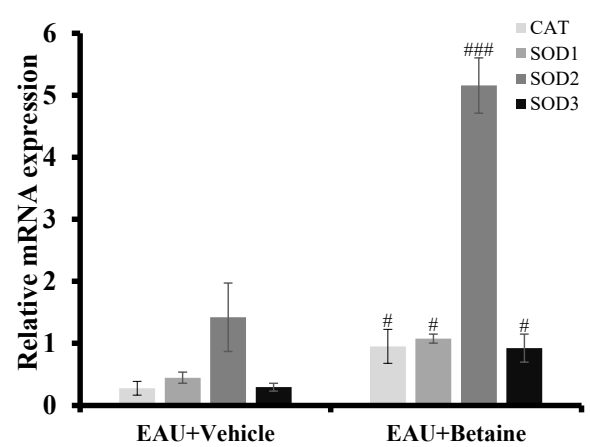

Fig. 5. The analysis of adhesion molecule, pro-inflammatory mediators, and anti-oxidant molecules after betaine treatment. (A) Although the existence of vascular cell adhesion molecule 1 (VCAM1), one of the representative adhesion molecule, was kept in higher expression level in EAU+Vehicle group, the betaine treatment inhibited to rising of the VCAM1. (B) Consequently, the Serpina3n, interleukin-1 $1 \beta$ (IL- $1 \beta$ ), tumor necrosis factor- $\alpha$ (TNF- $\alpha$ ) and cyclooxygenase-2 (COX-2) were showed analogous to that of VCAM1 inclination, but not in inducible nitric oxide synthase (iNOS). (C) The mRNA levels of the antioxidant enzymes catalase (CAT), superoxide dismutase (SOD) 1, SOD2, and SOD3 increased in the EAU+Betaine group. ${ }^{*} \mathrm{p}<0.05$; ${ }^{* *} \mathrm{p}<0.01$ vs. normal control; ${ }^{\dagger} \mathrm{p}<0.05$ vs. CFA; ${ }^{*} \mathrm{p}<0.05 ;{ }^{\# *} \mathrm{p}<0.01 ;{ }^{* \# *} \mathrm{p}<0.001$ vs. EAU+Vehicle.

A

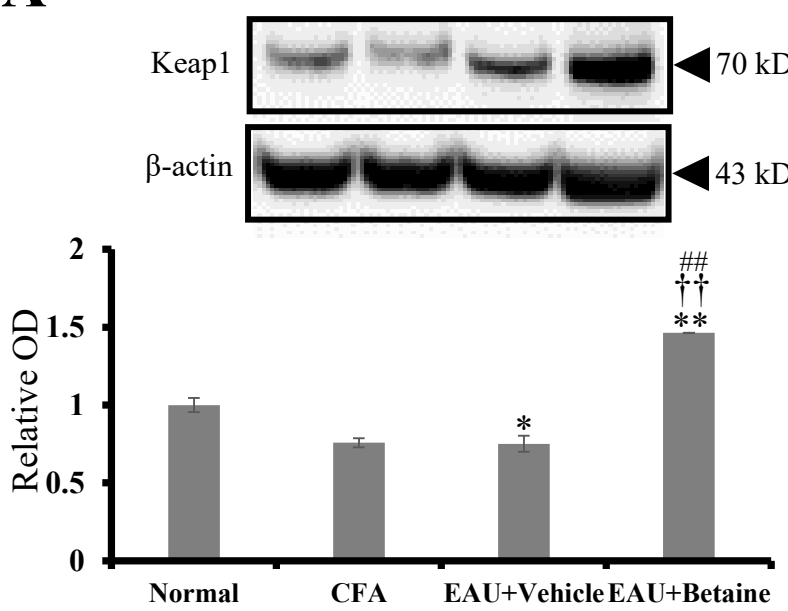

B

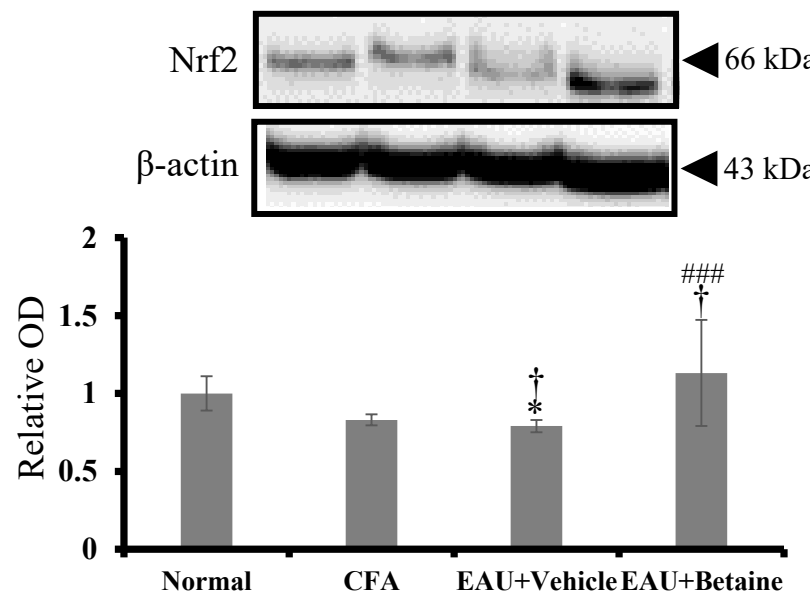

Fig. 6. Western blot analysis of the eyes in EAU-induced rats. The expression levels of Kelch-like ECH-associated protein 1 (Keap1) (A) and Nuclear factor erythroid-2-related factor $2(\mathrm{Nrf} 2)(\mathrm{B})$ was promoted by the betaine treatment after the induction of EAU. ${ }^{*} \mathrm{p}<0.05$; ${ }^{* *} \mathrm{p}<0.01$ vs. normal control; ${ }^{\dagger} \mathrm{p}<0.05 ;{ }^{\dagger \dagger} \mathrm{p}<0.01$ vs. CFA; ${ }^{* \#} \mathrm{p}<0.01 ;{ }^{\# \# *} \mathrm{p}<0.001$ vs. EAU+Vehicle.

ase-2 (COX-2) as pro-inflammatory mediators, were assessed to confirm the inflammatory condition (Fig. 5B). The mRNA levels of Serpina3n, IL-1 $\beta$, TNF- $\alpha$, COX-2 were significantly downregulated in the $\mathrm{EAU}+\mathrm{Betaine}$ group compared with that of vehicletreated EAU group (Fig. 5B). These results indicate that the betaine treatment suppressed the upregulation of pro-inflammatory mediators.

\section{Betaine upregulated the antioxidant enzymes catalase (CAT) and SOD in EAU}

The study of oxidative damage levels in the serum prompted us to investigate the antioxidant response status of antioxidant enzymes, including CAT, SOD1, SOD2 and SOD3 (Fig. 5C). We observed significantly upregulated expression levels of CAT, SOD1, SOD2 and SOD3 in the eyeballs of the EAU+Betaine group compared with the EAU+Vehicle group.

\section{Betaine activated the Keap1-Nrf2 pathway}

To support the anti-oxidative effect of betaine, the Keap 1$\mathrm{Nrf} 2$ pathway was examined (Fig. 6). The protein levels of Keap1 $(0.75 \pm 0.05$ fold changes, $p<0.05$, Fig. $6 \mathrm{~A})$ and $\mathrm{Nrf} 2(0.79 \pm 0.04$ fold changes, $\mathrm{p}<0.05$, Fig. $6 \mathrm{~B}$ ) in EAU+Vehicle group were suppressed 

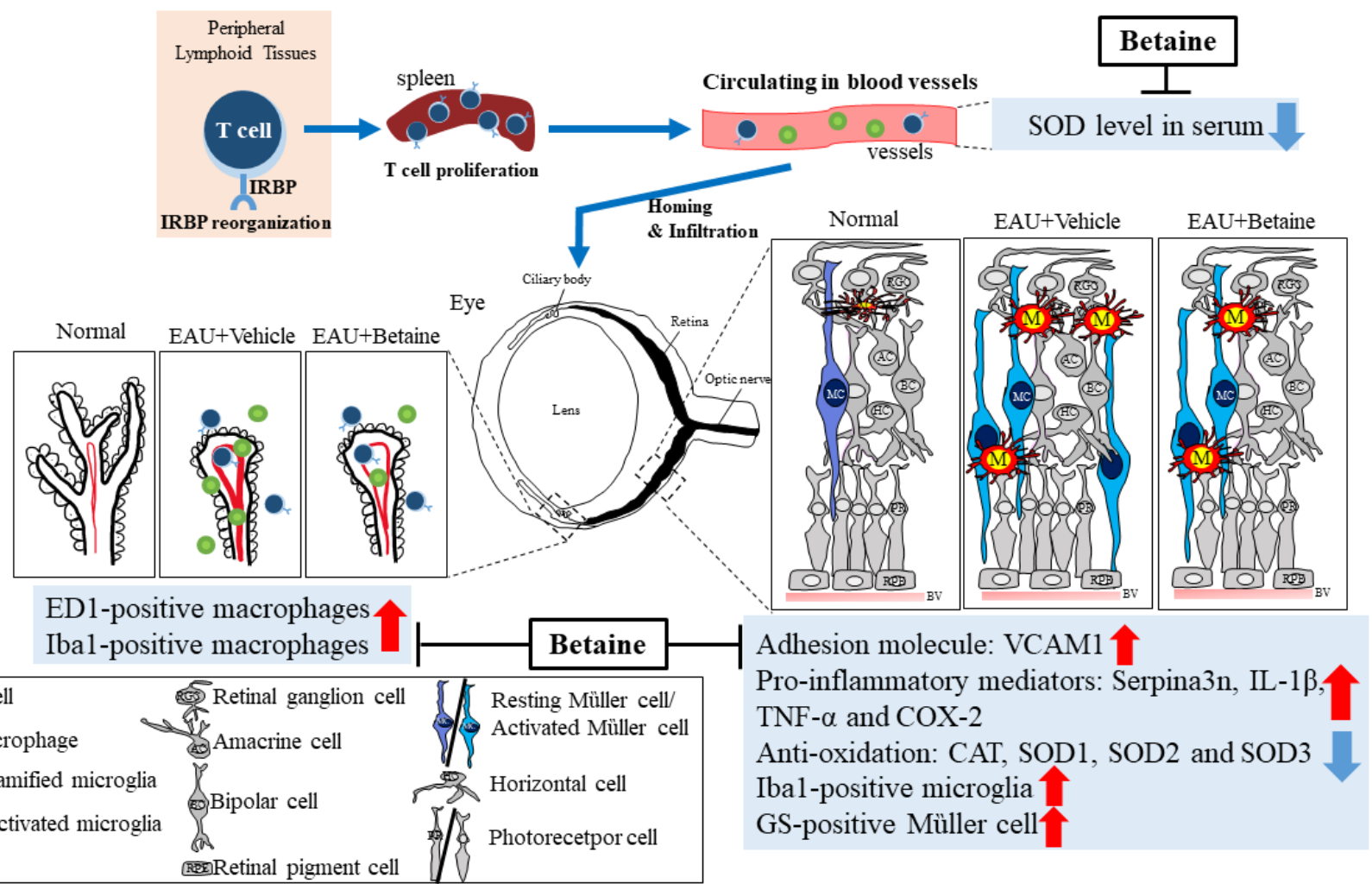

Fig. 7. Schematic illustration of the anti-inflammatory and anti-oxidant effects of betaine in EAU-induced rats. EAU was initiated by T cell proliferation induced by IRBP recognition. The IRBP-induced T cells circulated through the vessels and homed in on the eye as a target organ. The infiltration of Ibal-and ED1-positive macrophages was detected in the ciliary body. Inflammatory cell infiltration decreased in the betaine-treated group. Betaine treatment led to the downregulation of the levels of VCAM1, Serpina3n, IL-1 $\beta$, TNF- $\alpha$, COX-2, Ibal and GS and upregulation of CAT, SOD1, SOD2 and SOD3. Moreover, betaine treatment restored the blood SOD concentrations.

compared with the normal control. On the other hand, the Keap1 and Nrf2 showed either $1.46 \pm 0.00$ fold changes or $1.13 \pm 0.34$ fold changes than those of EAU+Vehicle group $(\mathrm{p}<0.01$ and $\mathrm{p}<0.001$, respectively).

\section{DISCUSSION}

This is the first study reporting that betaine mitigates the progression of EAU pathogenesis through anti-inflammatory and anti-oxidant effects, but not by suppressing $\mathrm{T}$ cell proliferation (Schematic illustration in Fig. 7).

The regulatory effect of betaine in autoimmune diseases as evidenced using EAU, a prototype of autoimmune disease, is thought to be due to the reduction of oxidative stress and pro-inflammatory mediators, but not $\mathrm{T}$ cell proliferation, by betaine [19]. Similarly, the present study revealed that betaine had little effect on $\mathrm{T}$ cell proliferation and the cytokine profile in the culture supernatant in an EAU model, suggesting that betaine does not influence the immune response of proliferation of autoimmune T cells in EAU.

The uvea is a target organ in EAU. The uvea and retina are im- munologically isolated organs without lymphatics [20]. The autoimmune $\mathrm{T}$ cells in EAU are invaded via a branch of the ciliary and ophthalmic arteries [21]. Oxidative stress is a critical signaling to the progression of inflammatory response and the increased reactive oxygen species causes endothelial dysfunction and tissue injury [22]. The disturbed endothelial cells are lead to promotion of passage of inflammatory cells and inflammatory molecules [22]. Inflammatory mediators and cells in the uvea are triggered to the retinal pigment epithelial cells, which disturb the junctions between rod and cone cells and pigmented epithelial cells, leading to a detached retina [23]. The ciliary body is an entry site for ocular inflammation. Typical retinal inflammation is involved in the activation of resident microglia and the infiltration of inflammatory cells because of the breakdown of the blood-retina barrier [24]. Under the neuropathological conditions, including brain tumors [25], axotomy [26] and virus infection [27], the activated macrophages and microglia was distinguished by Ibal. In addition, activated resident microglia are involved in the pathological changes occurring in retinal degenerative diseases and release inflammatory mediators that exacerbate the disease process [28]. 
These results suggest that betaine exerts anti-inflammatory effects in the uvea and ciliary body, the main targets of EAU, and may reduce oxidative stress in the serum. However, the precise mechanism remains to be studied.

Activated microglia are the main source of pro-inflammatory cytokines under retinal degenerative conditions [29]. Pro-inflammatory cytokines, including ILs and TNF, are strongly associated with ocular inflammation [30] and retinitis [6,29]. Besides the microglia, Müller cells are activated under all pathological events that occur in the retina [31]. Activated Müller cells are involved in the neuroinflammatory effect in the retina by synthesizing and releasing inflammation-related molecules [31]. We postulate that betaine mitigates the inflammatory response in EAU-induced rats by suppressing the activation of microglia and Müller cells.

The upregulation of VCAM1 is highly involved in the infiltration of inflammatory cells [32]. VCAM1 is expedited to CD4 T lymphocytes through cross-talk with late antigen-4 [33]. In addition, Serpina3n, an enzyme that initiates inflammation [34], has been detected in Müller cells, astrocytes and retinal pigment epithelia of light-damaged retinas [35] and has significantly variable levels in the $\mathrm{Nrl}^{-/-}$mouse retina with impaired cone cells [36]. Serpina3n is increased in EAU rats with severe retinal inflammation but decreased significantly in the betaine-treated EAU group. A similar finding has been reported for schizophrenia with neuroinflammation [34], as murine Serpina3n is an orthologue of human Serpina3 [37]. Furthermore, an increase in IL-1 $\beta$ was observed in high fructose-induced retinal injury [38]. We postulate that the anti-inflammatory effect of betaine is associated with the downregulation of VCAM1, Serpina3n and IL- $1 \beta$ in EAU-induced rats.

The Keap1-Nrf2 pathway is used to monitor the oxidative stress [39]. The betaine had known for an anti-oxidant molecule, which was associated with Keap1-Nrf2 pathway in acetaminophen induced acute liver injury model [40]. Additionally, the hepatic gene expression profiling was performed after the $3 \mathrm{H}$-1,2-dithiole3 -thione treatment, having the roles of enhancing the detoxification of carcinogens and protecting against neoplasia [41]. The result of this profiling was revealed that the Keap1-Nrf2 regulated nrf2-dependent 3H-1,2-dithiole-3-thione-inducible gene, including AF033381, as betaine homocysteine methyl transferase, was increased and involved to the detoxification and anti-oxidation [41]. The inflammatory response in EAU was induced by infiltration of inflammatory cells, such as T cell and macrophages [42], and production of oxidative stress, especially in photoreceptor mitochondria of early stage [43]. According to these results, the betaine treatment was a candidate to relieve the EAU-induced tissue damage by modulation of Keap1-Nrf2 pathway, as a key pathway to regulation of oxidative stress.
The antioxidant effect of betaine has been widely evaluated in radical-induced injury models [44]. In the levodopa-induced oxidative-damage brain, betaine was enhanced to the levels of CAT and SOD, which are representative antioxidant enzymes [44]. SOD1, SOD2 and SOD3 are activated by different mechanisms and are localized in the cytoplasm, mitochondria, and extracellular matrix, respectively [45]. Betaine, as an anti-oxidative molecule, is involved in reducing of the oxidative damage [46]. The reduced oxidative stress was extended to resolving the inflammation, indicated by Ibal-positive macrophages/microglia in many diseases, including Alzheimer's disease, Parkinson disease and multiple sclerosis [47]. The betaine treatment was upregulated to the mRNA levels of oxidative stress marker, compared with those in the EAU+Vehicle group. This result implies that the betaine treatment reduced oxidative stress in the circulatory system without interfering with $\mathrm{T}$ cell proliferation in the immune organs of the rat EAU model.

Collectively, the present study suggests that betaine can mitigate inflammation in the retinas and ciliary bodies of EAU-induced rats, possibly through anti-oxidation and anti-inflammation mechanisms.

\section{ACKNOWLEDGEMENTS}

The experiments in the present study was designed by TS and performed by YC, KJ, and HK. Figure plates were prepared by YC, $\mathrm{KJ}$, amd HK. The manusctipr was written by YC and TS, and all authors (KJ, HK, JC, MA, YJ, HK, CM, HM, AT, JK, and TS) read and approved the final manuscript. This research was supported by the National Research Foundation of Korea (Grant number: NRF 2019R1A2C1087753).

\section{REFERENCES}

1. Forrester JV, Kuffova L, Dick AD (2018) Autoimmunity, autoinflammation, and infection in uveitis. Am J Ophthalmol 189:77-85

2. Kurschus FC (2015) T cell mediated pathogenesis in EAE: molecular mechanisms. Biomed J 38:183-193.

3. Tajiri K, Shimojo N, Sakai S, Machino-Ohtsuka T, ImanakaYoshida K, Hiroe M, Tsujimura Y, Kimura T, Sato A, Yasutomi Y, Aonuma K (2013) Pitavastatin regulates helper T-cell differentiation and ameliorates autoimmune myocarditis in mice. Cardiovasc Drugs Ther 27:413-424.

4. Shen D, Chu F, Lang Y, Geng Y, Zheng X, Zhu J, Liu K (2018) Beneficial or harmful role of macrophages in Guillain-Barré syndrome and experimental autoimmune neuritis. Mediators 
Inflamm 2018:4286364.

5. Zhang Z, Liu Q, Leskov KS, Wu X, Duan J, Zhang GL, Hall M, Rosenbaum JT (2013) Roscovitine suppresses CD4+ T cells and T cell-mediated experimental uveitis. PLoS One 8:e81154.

6. Lee RW, Nicholson LB, Sen HN, Chan CC, Wei L, Nussenblatt RB, Dick AD (2014) Autoimmune and autoinflammatory mechanisms in uveitis. Semin Immunopathol 36:581-594.

7. Saraswathy S, Rao NA (2008) Photoreceptor mitochondrial oxidative stress in experimental autoimmune uveitis. Ophthalmic Res 40:160-164.

8. Zhao G, He F, Wu C, Li P, Li N, Deng J, Zhu G, Ren W, Peng Y (2018) Betaine in inflammation: mechanistic aspects and applications. Front Immunol 9:1070.

9. Go EK, Jung KJ, Kim JY, Yu BP, Chung HY (2005) Betaine suppresses proinflammatory signaling during aging: the involvement of nuclear factor-kappaB via nuclear factor-inducing kinase/IkappaB kinase and mitogen-activated protein kinases. J Gerontol A Biol Sci Med Sci 60:1252-1264.

10. Detopoulou P, Panagiotakos DB, Antonopoulou S, Pitsavos C, Stefanadis C (2008) Dietary choline and betaine intakes in relation to concentrations of inflammatory markers in healthy adults: the ATTICA study. Am J Clin Nutr 87:424-430.

11. Kim DH, Sung B, Kang YJ, Jang JY, Hwang SY, Lee Y, Kim M, Im E, Yoon JH, Kim CM, Chung HY, Kim ND (2014) Antiinflammatory effects of betaine on AOM/DSS-induced colon tumorigenesis in ICR male mice. Int J Oncol 45:1250-1256.

12. Kim YG, Lim HH, Lee SH, Shin MS, Kim CJ, Yang HJ (2015) Betaine inhibits vascularization via suppression of Akt in the retinas of streptozotocin-induced hyperglycemic rats. Mol Med Rep 12:1639-1644.

13. Chang RC, So KF (2008) Use of anti-aging herbal medicine, Lycium barbarum, against aging-associated diseases. What do we know so far? Cell Mol Neurobiol 28:643-652.

14. Kim SJ, Lee L, Kim JH, Lee TH, Shim I (2013) Antidepressantlike effects of lycii radicis cortex and betaine in the forced swimming test in rats. Biomol Ther (Seoul) 21:79-83.

15. Kim H, Moon C, Park EJ, Jee Y, Ahn M, Wie MB, Shin T (2010) Amelioration of experimental autoimmune encephalomyelitis in Lewis rats treated with fucoidan. Phytother Res 24:399403.

16. Choi Y, Kim J, Ahn M, Shin T (2020) Upregulation of periostin in MOG-induced experimental autoimmune encephalomyelitis in mice. Neurosci Lett 715:134558.

17. Caspi RR (2003) Experimental autoimmune uveoretinitis in the rat and mouse. Curr Protoc Immunol Chapter 15:Unit 15.6 .
18. Delamere NA (2005) Ciliary body and ciliary epithelium. Adv Organ Biol 10:127-148.

19. Yang C, Lai W, Zhou J, Zheng X, Cai Y, Yang W, Xie S, Gao Y, Du C (2018) Betaine ameliorates experimental autoimmune encephalomyelitis by inhibiting dendritic cell-derived IL-6 production and Th17 differentiation. J Immunol 200:13161324.

20. Zhou R, Caspi RR (2010) Ocular immune privilege. F1000 Biol Rep 2:3.

21. Hayreh SS (2006) Orbital vascular anatomy. Eye (Lond) 20:1130-1144.

22. Mittal M, Siddiqui MR, Tran K, Reddy SP, Malik AB (2014) Reactive oxygen species in inflammation and tissue injury. Antioxid Redox Signal 20:1126-1167.

23. Del Amo EM, Rimpelä AK, Heikkinen E, Kari OK, Ramsay E, Lajunen T, Schmitt M, Pelkonen L, Bhattacharya M, Richardson D, Subrizi A, Turunen T, Reinisalo M, Itkonen J, Toropainen E, Casteleijn M, Kidron H, Antopolsky M, Vellonen KS, Ruponen M, Urtti A (2017) Pharmacokinetic aspects of retinal drug delivery. Prog Retin Eye Res 57:134-185.

24. Crane IJ, Liversidge J (2008) Mechanisms of leukocyte migration across the blood-retina barrier. Semin Immunopathol 30:165-177.

25. Tran CT, Wolz P, Egensperger R, Kösel S, Imai Y, Bise K, Kohsaka S, Mehraein P, Graeber MB (1998) Differential expression of MHC class II molecules by microglia and neoplastic astroglia: relevance for the escape of astrocytoma cells from immune surveillance. Neuropathol Appl Neurobiol 24:293301.

26. Graeber MB, López-Redondo F, Ikoma E, Ishikawa M, Imai Y, Nakajima K, Kreutzberg GW, Kohsaka S (1998) The microglia/macrophage response in the neonatal rat facial nucleus following axotomy. Brain Res 813:241-253.

27. Mori I, Imai Y, Kohsaka S, Kimura Y (2000) Upregulated expression of Ibal molecules in the central nervous system of mice in response to neurovirulent influenza A virus infection. Microbiol Immunol 44:729-735.

28. Rashid K, Akhtar-Schaefer I, Langmann T (2019) Microglia in retinal degeneration. Front Immunol 10:1975.

29. Langmann T (2007) Microglia activation in retinal degeneration. J Leukoc Biol 81:1345-1351.

30. Luna JD, Chan CC, Derevjanik NL, Mahlow J, Chiu C, Peng B, Tobe T, Campochiaro PA, Vinores SA (1997) Blood-retinal barrier (BRB) breakdown in experimental autoimmune uveoretinitis: comparison with vascular endothelial growth factor, tumor necrosis factor alpha, and interleukin-1betamediated breakdown. J Neurosci Res 49:268-280. 
31. Kumar A, Pandey RK, Miller LJ, Singh PK, Kanwar M (2013) Muller glia in retinal innate immunity: a perspective on their roles in endophthalmitis. Crit Rev Immunol 33:119-135.

32. Wu H, Deng R, Chen X, Wong WC, Chen H, Gao L, Nie Y, Wu W, Shen J (2016) Caveolin-1 is critical for lymphocyte trafficking into central nervous system during experimental autoimmune encephalomyelitis. J Neurosci 36:5193-5199.

33. Kim SHJ, Hammer DA (2019) Integrin crosstalk allows CD4+ T lymphocytes to continue migrating in the upstream direction after flow. Integr Biol (Camb) 11:384-393.

34. Fillman SG, Sinclair D, Fung SJ, Webster MJ, Shannon Weickert C (2014) Markers of inflammation and stress distinguish subsets of individuals with schizophrenia and bipolar disorder. Transl Psychiatry 4:e365.

35. Rattner A, Nathans J (2005) The genomic response to retinal disease and injury: evidence for endothelin signaling from photoreceptors to glia. J Neurosci 25:4540-4549.

36. Roger JE, Ranganath K, Zhao L, Cojocaru RI, Brooks M, Gotoh N, Veleri S, Hiriyanna A, Rachel RA, Campos MM, Fariss RN, Wong WT, Swaroop A (2012) Preservation of cone photoreceptors after a rapid yet transient degeneration and remodeling in cone-only Nrl-/- mouse retina. J Neurosci 32:528-541.

37. Aslam MS, Yuan L (2020) Serpina3n: potential drug and challenges, mini review. J Drug Target 28:368-378.

38. Fan CY, Wang MX, Ge CX, Wang X, Li JM, Kong LD (2014) Betaine supplementation protects against high-fructoseinduced renal injury in rats. J Nutr Biochem 25:353-362.

39. Yu C, Xiao JH (2021) The Keap1-Nrf2 system: a mediator between oxidative stress and aging. Oxid Med Cell Longev 2021:6635460.

40. Khodayar MJ, Kalantari H, Khorsandi L, Rashno M, Zeidooni
L (2020) Upregulation of Nrf2-related cytoprotective genes expression by acetaminophen-induced acute hepatotoxicity in mice and the protective role of betaine. Hum Exp Toxicol 39:948-959.

41. Kwak MK, Wakabayashi N, Itoh K, Motohashi H, Yamamoto M, Kensler TW (2003) Modulation of gene expression by cancer chemopreventive dithiolethiones through the Keap1Nrf2 pathway. Identification of novel gene clusters for cell survival. J Biol Chem 278:8135-8145.

42. Sonoda KH, Sasa Y, Qiao H, Tsutsumi C, Hisatomi T, Komiyama S, Kubota T, Sakamoto T, Kawano Y, Ishibashi T (2003) Immunoregulatory role of ocular macrophages: the macrophages produce RANTES to suppress experimental autoimmune uveitis. J Immunol 171:2652-2659.

43. Rajendram R, Saraswathy S, Rao NA (2007) Photoreceptor mitochondrial oxidative stress in early experimental autoimmune uveoretinitis. Br J Ophthalmol 91:531-537.

44. Alirezaei M, Khoshdel Z, Dezfoulian O, Rashidipour M, Taghadosi V (2015) Beneficial antioxidant properties of betaine against oxidative stress mediated by levodopa/benserazide in the brain of rats. J Physiol Sci 65:243-252.

45. Fukai T, Ushio-Fukai M (2011) Superoxide dismutases: role in redox signaling, vascular function, and diseases. Antioxid Redox Signal 15:1583-1606.

46. Ganesan B, Anandan R, Lakshmanan PT (2011) Studies on the protective effects of betaine against oxidative damage during experimentally induced restraint stress in Wistar albino rats. Cell Stress Chaperones 16:641-652.

47. Popa-Wagner A, Mitran S, Sivanesan S, Chang E, Buga AM (2013) ROS and brain diseases: the good, the bad, and the ugly. Oxid Med Cell Longev 2013:963520. 